\title{
Performance of the LBNL FastCCD for the European XFEL
}

\author{
Friederike Januschek ${ }^{a}$, Ivana Klačkova ${ }^{a, b}$, Nord Andresen ${ }^{c}$, Peter Denes ${ }^{c}$, Steffen Hauf ${ }^{a}$, \\ John Joseph ${ }^{c}$, Markus Kuster ${ }^{a}$, Craig Tindall ${ }^{c}$ \\ ${ }^{a}$ European XFEL GmbH, Holzkoppel 4, 22869 Schenefeld, Germany \\ ${ }^{b}$ Slovak University of Technology, Faculty of Electrical Engineering and Information Technology, Ilkovičova 3, Bratislava, SK-812 19, Slovak Republic \\ ${ }^{c}$ Lawrence Berkeley National Laboratory, 1 Cyclotron Road, Berkeley, CA 94720, U.S.A.
}

\begin{abstract}
The European X-ray Free Electron Laser (XFEL.EU) is currently being commissioned in Schenefeld, Germany. From 2017 onwards it will provide spatially coherent $\mathrm{X}$-rays of energies between $0.25 \mathrm{keV}$ and $25 \mathrm{keV}$ with a unique timing structure. One of the detectors foreseen at XFEL.EU for the soft $X$-ray regime (energies below $6 \mathrm{keV}$ ) is a quasi column-parallel readout FastCCD developed by Lawrence Berkeley National Lab (LBNL) specifically for the XFEL.EU requirements. Its sensor has $1920 \times 960$ pixels of $30 \mu \mathrm{m} \times 30 \mu \mathrm{m}$ size with a beam hole in the middle of the sensor. The camera can be operated in full frame and frame store mode. With the FastCCD a frame rate of up to 120 fps can be achieved, but at XFEL.EU the camera settings are optimized for the $10 \mathrm{~Hz}$ XFEL bunch-mode. The detector has been delivered to XFEL.EU. Results of the performance tests and calibration done using the XFEL.EU detector calibration infrastructure are presented quantifying noise level, gain and energy resolution.

Index Terms-X-ray detectors, CCD, FEL
\end{abstract}

\section{INTRODUCTION}

From 2017 onwards the European X-ray Free Electron Laser (XFEL.EU) [1], which is currently being commissioned in Schenefeld, Germany, will provide spatially coherent X-rays with a unique timing structure: there will be up to 2700 pulses, with a $4.5 \mathrm{MHz}$ repetition rate, 10 times per second at very high photon fluxes up to $10^{13}$ photons per pulse [2]. For the planned energy range between $0.25 \mathrm{keV}$ and $25 \mathrm{keV}$ several dedicated detectors are foreseen [3]. One of the detectors for the soft X-ray regime will be a FastCCD with a quasi columnparallel readout, which has been developed at the Lawrence Berkeley National Lab (LBNL) [4].

\section{THE LBNL FASTCCD}

The 1k Frame Store (FS) FastCCD Sensor System developed at LBNL is a soft X-ray detector including a readout system optimized for experiments at advanced light sources. It is designed for energies of $0.25 \mathrm{keV}<E_{\gamma}<6 \mathrm{keV}$. The main components of the system are an in-vacuum camera head shown in Figure 1, a camera interface electronics board and an ATCA based readout and data processing subsystem [5]. The full camera head assembly includes the three separate electronics subassemblies and a cooling system to cool the camera to operating temperature of approximately $-57^{\circ} \mathrm{C}$. For increased speed the readout is done almost column parallel with a ten column multiplexed readout.

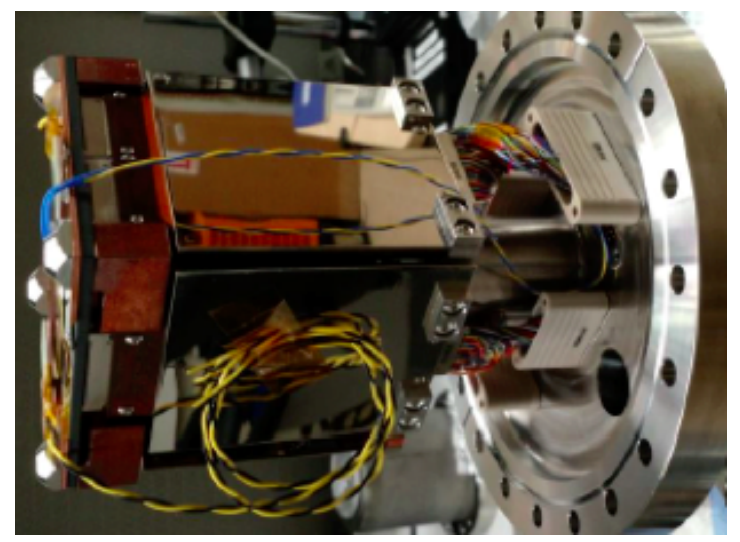

Fig. 1: The assembled FastCCD camera for XFEL.EU.

TABLE I: Technical specifications of the FastCCD detector developed at LBNL for XFEL.EU.

$\begin{array}{ll}\text { Properties } & \text { FastCCD } \\ \text { Photon energy range } & 0.25 \mathrm{keV}-6 \mathrm{keV} \\ \text { Pixel size } & 30 \mu \mathrm{m} \times 30 \mu \mathrm{m} \\ \text { Sensor size } & 1920 \times 960 \text { pixels } \\ \text { Sensor thickness } & 200 \mu \mathrm{m} \\ \text { Dynamic range } & 10^{3} \text { above } 0.5 \mathrm{keV} \\ \text { Beam hole size } & 1.8 \mathrm{~mm} \\ \text { Speed } & 60 \mathrm{fps}(\mathrm{FF}), 120 \mathrm{fps}(\mathrm{FS}) \\ \text { Quantum efficiency } & \geq 94 \% \text { for } E_{\gamma}>1 \mathrm{keV} \\ \text { Vacuum operation } & 10^{-7}-10^{-6} \text { mbar }\end{array}$

The detector manufactured for XFEL.EU has a sensor consisting of $1920 \times 960$ pixels of a size of $30 \mu \mathrm{m} \times 30 \mu \mathrm{m}$. Two operating modes are implemented: one using the entire sensor area for imaging (full-frame mode), the second using only the central $960 \times 960$ pixels for imaging and the remainder as a frame-store area. The detector specifications are given in Table I. The maximum achievable frame rate is 120 frames per second (fps) in frame-store mode and $60 \mathrm{fps}$ in full-frame mode. At XFEL.EU the camera will mainly be operated at $10 \mathrm{~Hz}$, corresponding to the pulse train rate of the FEL.

As the intensity of the XFEL.EU beam at the detector may be very high, a central hole for the beam to pass through is required. This hole of the size $1.8 \mathrm{~mm}$ was laser-drilled after the sensor had been fabricated, bonded and tested. During the drilling the sensor was protected by a thin layer of First Contact $^{\mathrm{TM}}$ cleaner. 


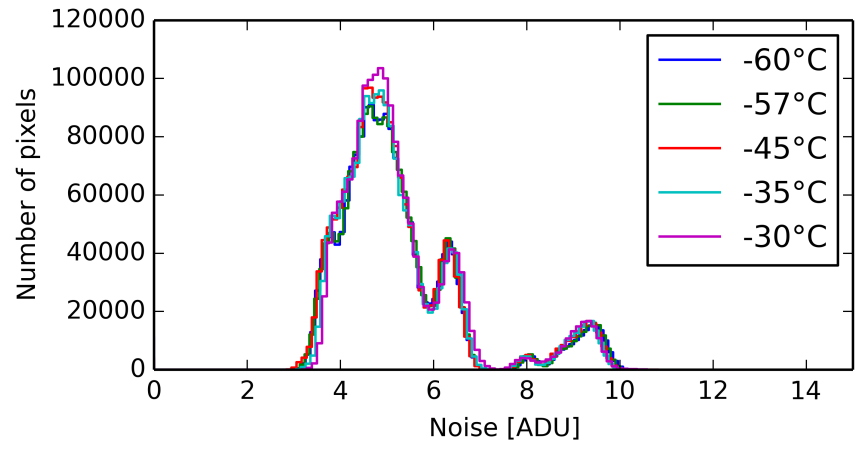

Fig. 2: Histogram showing the noise for the FastCCD pixels at different temperatures.

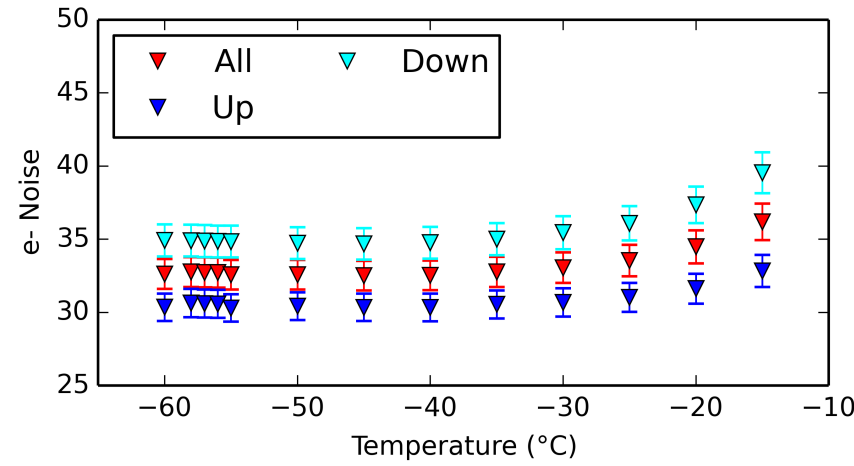

Fig. 3: Dependance on sensor temperature of the average noise for the whole sensor (red), the upper hemisphere (blue) and the lower hemisphere (cyan).

\section{Performance of the FastCCD}

First performance tests have been done showing good performance also after the $1.8 \mathrm{~mm}$ hole was cut in the sensor.

\section{A. Dark measurements}

The RMS noise level was measured to be $5.37 \pm 1.41$ ADU counts and is depicted in the histogram shown in Figure 2. Given the detector settings this is equivalent to $32.7 \pm 8.5 \mathrm{e}^{-}$. The noise and offset values were found to be stable within about $0.5 \%$ between $-60^{\circ} \mathrm{C}$ and $-30^{\circ} \mathrm{C}$ and then start increasing with rising temperature as expected (see Figure 3 ).

\section{B. Measurements with Fe-55 illumination}

Initial estimates of the detector response have been done by illuminating the sensor with a $1.1 \mathrm{GBq} F-55$ source using the XFEL.EU detector calibration laboratory [6].

Figure 4 shows an energy spectrum of 1-pixel single events (a single pixel having a signal above $5 \sigma_{\text {noise }}$, all neighboring pixels showing no signal, i.e. counts below $3 \sigma_{\text {noise }}$ ). The Mn $K_{\alpha}$ is clearly visible and its position as well as the position of the $\operatorname{Mn} K_{\beta}$ have been fitted to a 5-gaussian model. The fit to the position of these peaks yields a gain estimate of $22.26 \pm 0.2 \mathrm{eV} / \mathrm{ADU}$, which is in line with expectations. Note that the data from which the spectrum was produced has not yet been CTI or relative gain corrected. Nevertheless, an energy resolution of $\sigma=262 \pm 4 \mathrm{eV}$ can be observed,

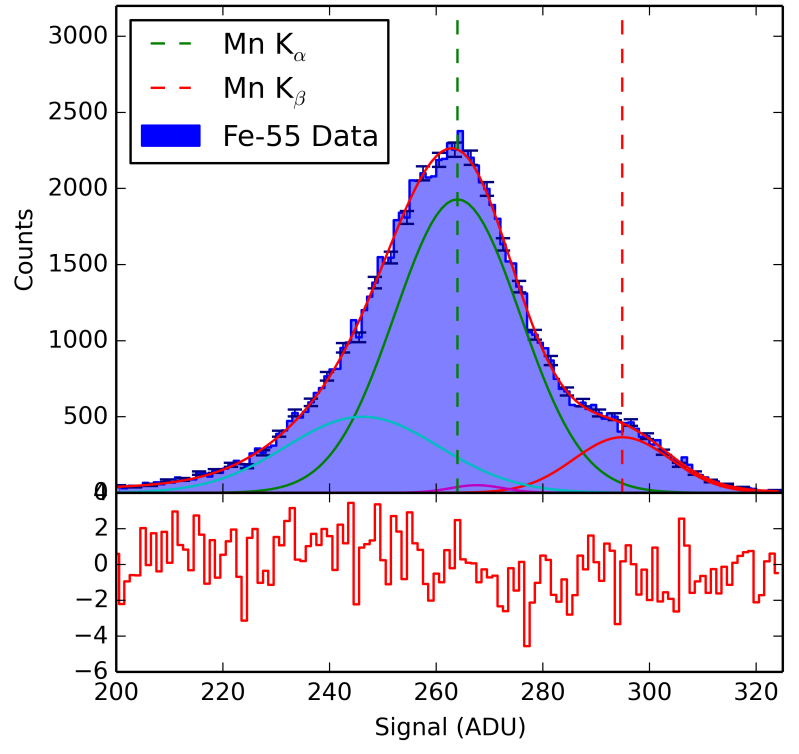

Fig. 4: The measured energy spectrum of an Fe-55 source is shown, taking only single-pixel events into account. The Mn $K_{\alpha}$ peak is clearly visible. The fit to $M n K_{\alpha}$ and $M n K_{\beta}$ and their positions are indicated by lines.

corresponding to a peak separation of $3.9 \sigma$ at the lower energy range of $1 \mathrm{keV}$, which is visualized in Figure 5 . The device thus offers reasonable performance for operation in a photonintegrating mode in these energy regimes.

\section{OUTLOOK}

With CTI and relative gain corrections the energy resolution has been improved to up to $\sigma=174 \pm 17 \mathrm{eV}$ for part of the detector. These corrections are currently limited by a synchronization issue in the firmware of the FastCCD. With a firmware update imminent, and further optimizations at LBNL (including an exchange of a noisy digitizer board), performance tests and calibration will continue in the XFEL.EU detector calibration laboratory. Using a pulsed X-ray source is planned, aiming at a calibration that allows an improved energy resolution.

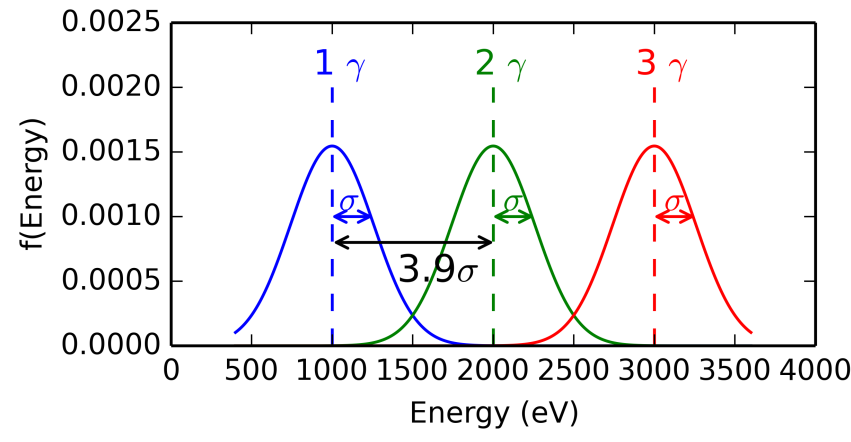

Fig. 5: Separation power of the FastCCD for photons at $1 \mathrm{keV}$. The predicted distributions of $1 \gamma, 2 \gamma$ and $3 \gamma$ events are shown and their separation of $3.9 \sigma$, i.e. an overlap of $2.6 \%$ of the event distributions. 
Afterwards, the FastCCD is to be integrated in the beam line of the Spectroscopy and Coherent Scattering [7] (SCS) experiment at XFEL.EU. There it will be utilized for singleshot non-linear imaging experiments and as a secondary diffraction plane for time-resolved holography together with the DSSC detector [8].

\section{CONCLUSiON}

Results of the performance tests for the LBNL FastCCD developed for the European XFEL have been presented. The RMS noise level is $32.7 \pm 8.5 \mathrm{e}^{-}$. With Fe-55 the energy resolution was measured to be $\sigma=262 \pm 4 \mathrm{eV}$. This is equivalent to a projected separation power of $3.9 \sigma$ at $1 \mathrm{keV}$. A further performance improvement is expected after replacement of a noisy digitizer board as well as optimization of the firmware and subsequently a full set of corrections including CTI and relative gain corrections.

\section{REFERENCES}

[1] M. Altarelli. The European X-ray free-electron laser. Technical Report July, European XFEL GmbH, 2006.

[2] Th. Tschentscher. Layout of the X-Ray Systems at the European XFEL. Technical Report April, European XFEL GmbH, 2011.

[3] M. Kuster, et al. Detectors and Calibration Concept for the European XFEL. Synchrotron Radiation News, volume 27(4):35-38, 2014.

[4] P. Denes, et al. A fast, direct X-ray detection charge-coupled device Review of Scientific Instruments, 80(083302), 2009.

[5] Patrick McVittie, et al. A Readout System for High-Speed CCD Cameras Based on Advanced Telecommunications Computing Architecture. In IEEE Nuclear Science Symposium Conference Record, pages 1623 -1625, 2012.

[6] Jolanta Sztuk-Dambietz, et al. Status of detector development for the European XFEL. In SPIE Proceedings, Advances in X-ray Free-Electron Lasers II: Instrumentation, volume 8778, 2013.

[7] A. Scherz, et al. Conceptual Design Report: Scientific Instrument Spectroscopy and Coherent Scattering (SCS). Technical report, European XFEL GmbH, 2013.

[8] Matteo Porro, et al. Development of the DEPFET sensor with signal compression: A large format X-ray imager with mega-frame readout capability for the European XFEL. IEEE Transactions on Nuclear Science, 59(6):3339-3351, 2012. 